\title{
Xuemaitong granules attenuate carotid atherosclerosis by decreasing the expression of CD14+CD16+ monocytes, IL-6, TNF- $\alpha$, and hsCRP
}

\author{
Q. Zhang ${ }^{1 *}$, G. Qian ${ }^{2 *}$ and Z. Ding ${ }^{2}$ \\ ${ }^{1}$ Department of Cardiology, \\ Changzhou Traditional Chinese Medicine Hospital Affiliated with Nanjing \\ Traditional Chinese Medicine University, Changzhou, China \\ ${ }^{2}$ Department of Clinical Laboratory, \\ Changzhou Traditional Chinese Medicine Hospital Affiliated with Nanjing \\ Traditional Chinese Medicine University, Changzhou, China \\ *These authors contributed equally to this study. \\ Corresponding author: Q. Zhang \\ E-mail: czzyyzhangqi@126.com
}

Genet. Mol. Res. 13 (3): 7519-7527 (2014)

Received July 25, 2013

Accepted March 11, 2014

Published September 12, 2014

DOI http://dx.doi.org/10.4238/2014.September.12.19

\begin{abstract}
Carotid atherosclerosis (CAS) has been extensively studied because its position can be easily observed. Our objective was to investigate the effects of Xuemaitong granules on the generation and activation of $\mathrm{CD} 14+\mathrm{CD} 16+$ monocytes on the inflammatory reaction in CAS patients. In this study, 22 male apolipoprotein E (apoE)deficient mice were fed a high-fat diet for 13 weeks. After induction of an atherosclerotic plaque, the animals were randomly divided into the Xuemaitong granule group $(450.5 \mathrm{mg} / \mathrm{kg}$ via intragastric administration, $\mathrm{N}=11$ ) and the control group (equal volume saline
\end{abstract}


via intragastric administration, $\mathrm{N}=11$ ). Venous blood was obtained to analyze monocyte and CD14+CD16+ inflammatory monocyte levels, as well as interleukin (IL)-6, tumor necrosis factor (TNF)- $\alpha$, and highsensitivity C-reactive protein (hsCRP). For clinical studies, $100 \mathrm{CAS}$ patients received oral administration of Xuemaitong granules for 6 months. Monocytes, CD14+CD16+ inflammatory monocytes, and the inflammatory cytokines IL-6, TNF- $\alpha$, and hsCRP were analyzed. Compared with the control group, a remarkable decrease in the number of monocytes and CD14+CD16+ inflammatory monocytes as well as TNF- $\alpha$, hsCRP, and IL- 6 was noted in the Xuemaitong group. Compared with before treatment levels, the proportions of monocytes and their subsets of CD14+CD16+ inflammatory monocytes and the concentration of the inflammatory cytokines IL-6, TNF- $\alpha$, and hsCRP significantly decreased. Xuemaitong granules played a significant role in the anti-inflammatory reactions. In addition, the granules attenuated the expression of the $\mathrm{CD} 14+\mathrm{CD} 16+$ inflammatory monocytes, resulting in the downregulation of the cytokines IL-6, TNF- $\alpha$, and hsCRP.

Key words: Monocytes; Inflammatory cytokines; Traditional Chinese medicine

\section{INTRODUCTION}

Carotid atherosclerosis (CAS), a type of systematic atherosclerosis (AS), has been extensively studied because its position can be easily observed. Additionally, these studies play a vital role for research by directly guiding clinical practice. Since 2003, we have investigated the use of traditional Chinese medicine (TCM) in patients with CAS. According to our previous studies, patients administered Xuemaitong granules, a TCM believed to remove blood stasis and downgrade turbidity of the kidney, showed satisfactory clinical outcomes (Zhang et al., 2006, 2007). However, its mechanism is still not well defined. Currently, AS has been well accepted to be an inflammatory disease since the first report by Ross (1999), revelead that AS is characterized by high lipoprotein and cholesterol plasma concentrations.

Recently, the mechanism of the inflammatory reaction in patients with atherosclerotic cardiovascular disease has been extensively studied (Imanishi et al., 2010; Merino et al., 2011; Berg et al., 2012; Rogacev et al., 2012; Zawada et al., 2012), which may provide some new ideas for clinical diagnosis and treatment. Based on these studies, the CD14+CD16+ monocyte subpopulation is thought to be closely related to inflammation in AS patients. As Xuemaitong granules have anti-inflammation effects, we hypothesized that they would suppress the inflammatory reaction by attenuating the proliferation and activation of CD14+CD16+ monocytes. In this study, we investigated the effects of Xuemaitong granules on $\mathrm{CD} 14+\mathrm{CD} 16+$ monocytes and the inflammatory factors interleukin (IL)-6, tumor necrosis factor (TNF)- $\alpha$, and high-sensitivity C-reactive protein (hsCRP) in AS patients. 


\section{MATERIAL AND METHODS}

Twenty-two male apolipoprotein E (apoE)-knockout mice (8-week-old, body weight: 20-22 g) with a C57/B1 genetic background were purchased from the Nanjing Biomedical Research Institutes of Nanjing University (Changzhou, China). The animals were raised in the Laboratory Animal Center and fed a high-fat diet (21\% fat, $0.15 \%$ cholesterol, treated using 60 Co $\gamma$ rays). The animals were housed under controlled temperatures (22$24^{\circ} \mathrm{C}$ ) with a 12 -h light $/ 12$-h dark cycle and food and water. The raising condition was grade 2 and relative humidity was $50 \%$. After exposure to the diet for 13 weeks, AS plaques were formed. The mice were randomly assigned into 2 groups: the Xuemaitong granule group $(450.5 \mathrm{mg} / \mathrm{kg}$ via intragastric administration, $\mathrm{N}=11$ ) and the control group (equal volume saline via intragastric administration, $\mathrm{N}=11$ ).

No food and water was given $24 \mathrm{~h}$ before blood collection. Blood collection was performed from the orbital venous plexus. Next, $100 \mu \mathrm{L}$ whole blood was used to test the proportion of monocytes. The remaining whole blood was centrifuged to collect the serum. Serum was stored at $-70^{\circ} \mathrm{C}$.

One hundred CAS patients (male: 68, female: 32 , aged 50-85 years) who had received intervention of Xuemaitong granules in our hospital were enrolled in this study. Clear sclerosis and plaques were identified in carotid arteries by color Doppler imaging. All patients were willing to receive an oral dose of Xuemaitong granules (twice daily, $17.5 \mathrm{~g}$ each dose) for 6 months. Exclusion criteria included patients with acute infection, cancer, autoimmune diseases, and chronic liver disease. The proportion of CD14+CD16+ monocytes was detected, and the serum was separated from the blood sample. Samples were preserved at $-70^{\circ} \mathrm{C}$ for further study. All patients signed informed consent. This study was approved by the Ethics Committee of Changzhou TCM Hospital Affiliated with Nanjing TCM University.

\section{Determination of monocytes and subgroups}

To evaluate monocytes, $30 \mu \mathrm{L}$ preliminary mixed monoclonal antibodies (CD14FITC/CD16-PE/CD45-PerCP; BD Biosciences; Franklin Lakes, NJ, USA) were added to the test tube. Next, $100 \mu \mathrm{L}$ whole blood was added. After oscillating and blending, samples were incubated in the dark for $20 \mathrm{~min}$, after which $500 \mu \mathrm{L}$ hemolysin was added and the mixture was incubated for $8 \mathrm{~min}$. Next, $500 \mu \mathrm{L}$ phosphate-buffered saline (PBS) was added and the sample was blended for $10 \mathrm{~min}$. The samples were loaded into the machine, monocytes were selected on the 2-parameter charts of CD45 and SSC, and then 2-parameter charts of CD14 and CD16 were used to analyze the proportion of monocytes in total leukocytes, CD14+ CD16+ monocytes, and CD14+CD16- monocytes.

\section{Expression of IL-6, TNF- $\alpha$, and hsCRP in serum}

IL-6 and TNF- $\alpha$ levels were determined using enzyme-linked immunosorbent assay (ELISA) kits (R\&D Systems; Minneapolis, MN, USA). The tests were performed strictly according to the protocols provided by the manufacturer. The Array 360 system (Beckman Specific Protein Analyzer; Brea, CA, USA) was used for protein analysis. The level of hsCRP was detected using a scattering immunoturbidimetric assay. 


\section{Determination of the inflammatory factors IL-6 and TNF- $\alpha$ in the plaque}

Total RNA was extracted according to the TRIzol kit instructions (Invitrogen; Carlsbad, CA, USA). For reverse transcription, $1 \mu \mathrm{g}$ RNA and $1 \mu \mathrm{L} 0.5 \mu \mathrm{g} / \mu \mathrm{L}$ Oligo (dT) primer were added. The total volume of the transcription reaction was $12 \mu \mathrm{L}$. After blending, samples were incubated at $65^{\circ} \mathrm{C}$ for $5 \mathrm{~min}$. Next, $1 \mu \mathrm{L} 20 \mathrm{U} / \mu \mathrm{L}$ RiboLock Ribonuclease inhibitor (Thermo Fisher Scientific, Shanghai, China), $4 \mu \mathrm{L} 5 \mathrm{X}$ reaction buffer, and 2 $\mu \mathrm{L} 10 \mathrm{mM}$ dNTPs were added, and the reaction was incubated at $37^{\circ} \mathrm{C}$ for $5 \mathrm{~min}$. Finally, $1 \mu \mathrm{L} 200 \mathrm{U} / \mu \mathrm{L}$ RevertAid M-Mulv Reverse Transcriptase was added to a $20 \mu \mathrm{L}$ volume. The mixture was incubated at $42^{\circ} \mathrm{C}$ for $60 \mathrm{~min}$, followed by heating at $72{ }^{\circ} \mathrm{C}$ for $10 \mathrm{~min}$ to terminate the reaction.

\section{Real-time polymerase chain reaction (PCR)}

Real-time PCR was performed according to the method provided by Roche (Mannheim, Germany) in a $20-\mu \mathrm{L}$ volume using $1 \mu \mathrm{L}$ cDNA, $1 \mu \mathrm{L}$ forward primer, $1 \mu \mathrm{L}$ reverse primer (Table 1), and $10 \mu \mathrm{L}$ SYBGreen Mix. The thermal cycling conditions were as follows: Stage $1=1$ cycle, $5 \mathrm{~min}$ at $95^{\circ} \mathrm{C}$; Stage $2=45 \mathrm{cycles}, 20 \mathrm{~s}$ at $95^{\circ} \mathrm{C}, 20 \mathrm{~s}$ at $65^{\circ} \mathrm{C}$, and $20 \mathrm{~s}$ at $72^{\circ} \mathrm{C}$.

Table 1. Sequence of the primers for real-time PCR.

\begin{tabular}{lll}
\hline Item & Sequence of forward primer & Sequence of reverse primer \\
\hline GAPDH & 5'-TGCCCCCATGTTTGTGAT-3' & 5'-GTCTTCTGGGTGGCAGTGAT-3' \\
IL-6 & 5'-GAAATCGTGGAAATGAGAAAAGAG-3' & 5'-CCTGATTATATCCAGTTTGGTAGCA-3' \\
TNF- $\alpha$ & 5'-CCTCTTCTCATTCCTGCTTGTG-3' & 5'-CACTTGGTGGTTTGCTACGAC-3' \\
\hline
\end{tabular}

\section{Western blot analysis}

Western blot analysis was performed as previously described (Fulda et al., 2000). Briefly, tissues were homogenized in RIPA lysis buffer containing protease and phosphatase inhibitors. Proteins were separated by electrophoresis on a $10 \%$ sodium dodecyl sulfate-polyacrylamide gel electrophoresis (SDS-PAGE) and transferred to a Hybond-P polyvinylidene fluoride membrane. Subsequently, the membrane was blocked in 5\% nonfat milk and incubated with an IL- 6 or TNF- $\alpha$ primary antibody $\left(1: 1000\right.$ dilution) overnight at $4{ }^{\circ} \mathrm{C}$, followed by incubation with peroxidase-conjugated rabbit anti-goat secondary antibody (1:1000 dilution) for $1 \mathrm{~h}$ at room temperature. After washing with Tris-buffered saline containing Tween 20 (TBST), bound primary antibody was visualized using the Enhanced Chemiluminescence System from Amersham (Piscataway, NJ, USA) and exposed to film. The same membrane was probed for $\beta$-actin, which was used as a loading control.

\section{Statistical analysis}

All data are reported as means \pm standard deviation. The SPSS 11.5 software was used for the statistical analysis (SPSS, Inc.; Chicago, IL, USA). The Student $t$-test was performed for intergroup comparisons. $\mathrm{P}<0.05$ was considered to be statistically significant. 


\section{RESULTS}

In this study, we investigated the effects of Xuemaitong granules on the expression of GrlLow (equivalent to the CD14+CD16+ inflammation mononuclear cells). A significant difference was observed in the monocyte to leukocyte ratio in the Xuemaitong group compared to the control group $(921 \pm 92 / 104$ vs $698 \pm 71 / 104, \mathrm{P}<0.01)$. In addition, a statistical difference was noted in the Xuemaitong group in the GrlLow/monocyte ratio compared with that of the control group $(191 \pm 54 / 104$ vs $75 \pm 41 / 104, \mathrm{P}<0.01)$.

In the Xuemaitong group, the Grlhigh to leukocyte ratio was $730 \pm 98 / 104$, which showed no statistical difference compared with the control group $(634 \pm 117 / 104, \mathrm{P}>0.05$; Figure 1). The concentrations of the inflammatory factors IL-6, TNF- $\alpha$, and hsCRP were also determined. The results showed statistical differences between the control group and the Xuemaitong group (17.9 \pm 1.7 vs $11.3 \pm 0.9 \mathrm{ng} / \mathrm{L}, \mathrm{P}<0.05 ; 52.8 \pm 6.3$ vs $36.9 \pm 4.6 \mathrm{ng} / \mathrm{L}, \mathrm{P}<0.05 ; 933.6 \pm 148.5$ vs $781.4 \pm$ $95.9 \mathrm{ng} / \mathrm{L}$, respectively, $\mathrm{P}<0.05$; Figure 2). The levels of IL- 6 and TNF- $\alpha$ were determined using reverse transcription PCR and Western blot analysis and demonstrated a remarkable decrease in the Xuemaitong group compared with the control group (Figure 3).

A

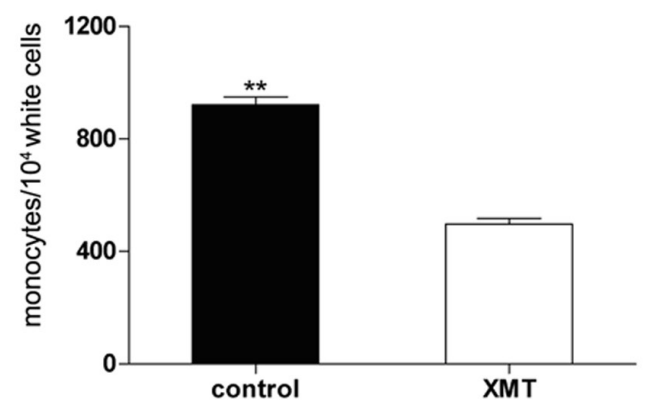

C

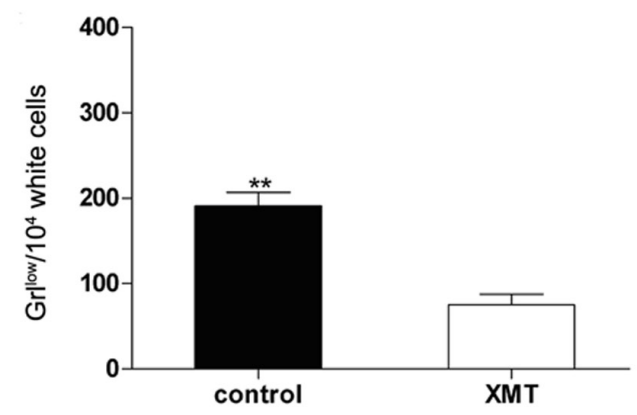

B

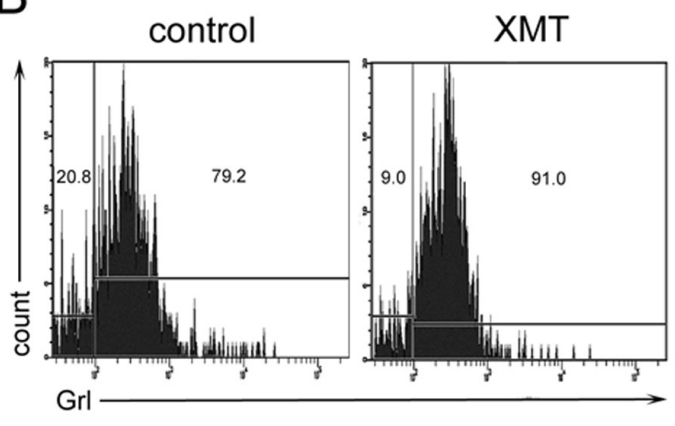

$\mathrm{D}$

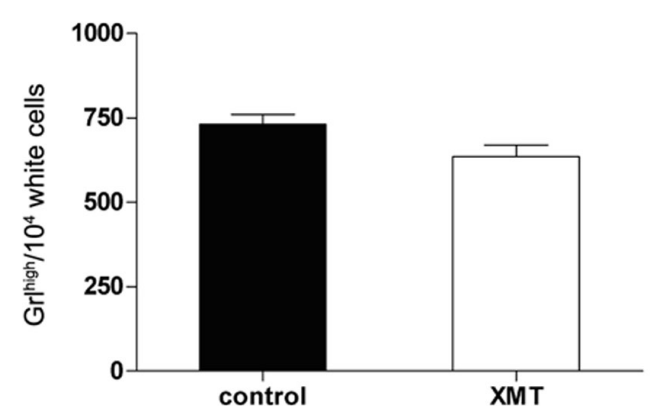

Figure 1. A. Monocytes/leukocytes in Xuemaitong group and control group in apoE knockout mice. B. Monocyte subsets GrlLow/monocytes in Xuemaitong group and control group in apoE knockout mice. C. Monocyte subsets GrlLow/leukocytes in Xuemaitong group and control group in apoE knockout mice; D. Monocyte subsets Grlhigh/ leukocytes in Xuemaitong group and control group in apoE knockout mice. Control $=$ control group. XMT $=$ Xuemaitong group. ${ }^{* *} \mathrm{P}<0.01$. 
A

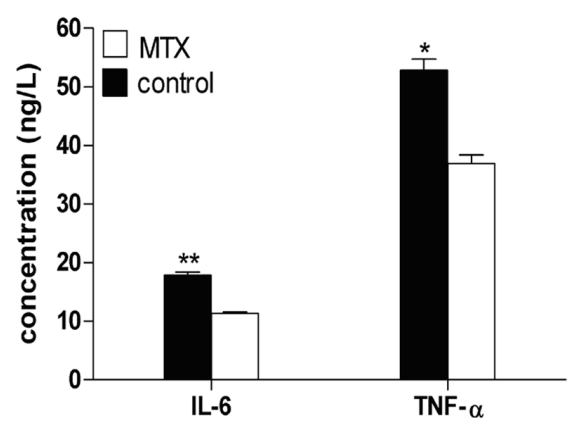

B

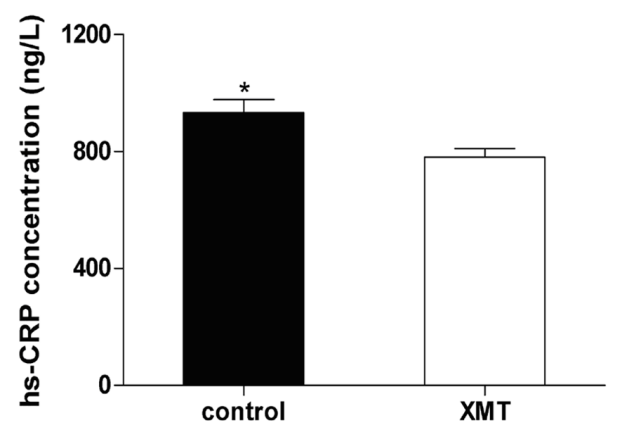

Figure 2. A. Serum concentrations of the IL- 6 and TNF- $\alpha$ in Xuemaitong granules group and control group in apoE knockout mice; B. Serum concentration of hs-CRP in Xuemaitong group and control group. Control = control group. XMT $=$ Xuemaitong group.

A

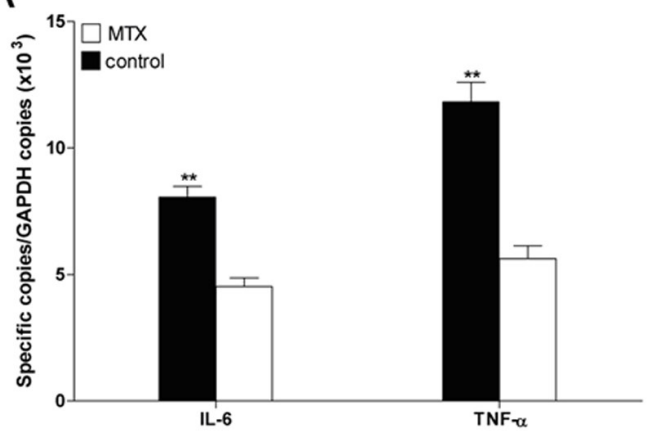

B

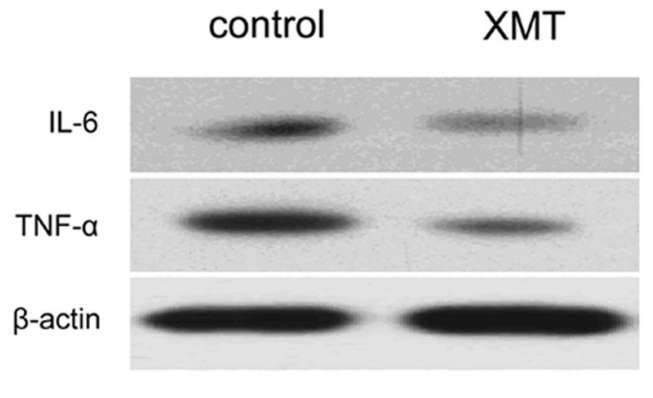

Figure 3. A. Expression of IL-6 and TNF- $\alpha$ in apoE knockout mice determined using Q-PCR; B. Expression of IL 6 and TNF- $\alpha$ in apoE knockout mice determined using Western blot. Control $=$ control group. XMT $=$ Xuemaitong group.

In our study, the effects of Xuemaitong on CD14+CD16 + monocytes were investigated. The results showed that the monocyte to leukocyte ratio was $738 \pm 174$ per $10^{4}$ cells before administration of Xuemaitong granules, while the ratio was $603 \pm 118$ per $10^{4}$ cells after treatment. A paired-samples Student $t$-test was performed, which showed a significant difference between groups $(\mathrm{P}<0.05)$. The $\mathrm{CD} 14+\mathrm{CD} 16+$ monocyte to leukocyte ratio was $69 \pm 25 / 104$ after treatment. A statistical difference was observed when compared with the baseline level $(131 \pm 45 / 104, \mathrm{P}<0.01)$. The CD14++CD16- monocyte to leukocytes ratio was $605 \pm 134 / 104$ and $545 \pm 121 / 104$, respectively ( $>0.05$, Figure 4$)$. Additionally, the concentration of the peripheral blood inflammatory factors IL-6, TNF- $\alpha$, and hsCRP in patients were determined. Statistical differences were observed for IL-6, TNF- $\alpha$, and hsCRP (40.0 $\pm 12.2 \mathrm{vs}$ $27.6 \pm 7.1 \mathrm{ng} / \mathrm{L} ; 71.6 \pm 17.3$ vs $54.0 \pm 14.9 \mathrm{ng} / \mathrm{L} ; 6891.5 \pm 1735.4$ vs $4848.9 \pm 1231.9 \mathrm{ng} / \mathrm{L}$, respectively; Figure 5). 
A

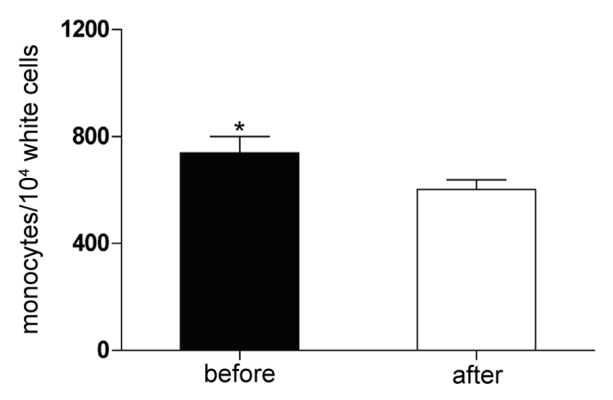

C

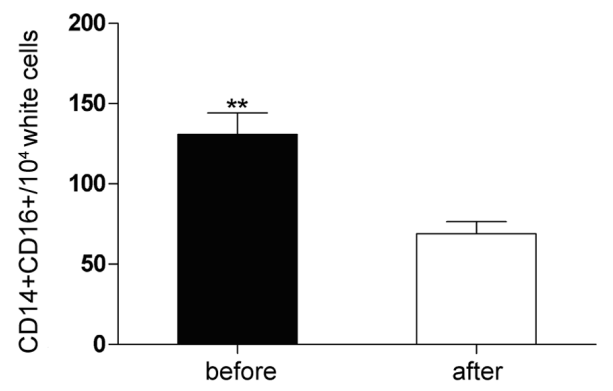

B

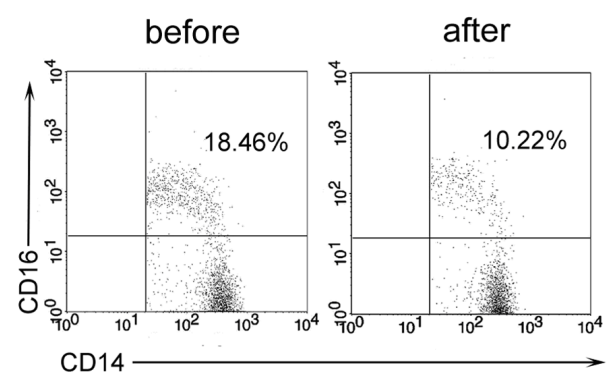

D

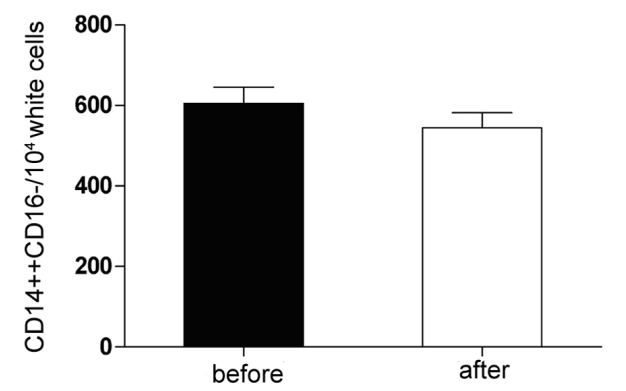

Figure 4. A. Monocytes/leukocytes in CAS patients; B. Ratio of CD14+CD16 + monocytes among the monocytes in CAS patients; C. CD14+CD16+ monocytes/leukocytes in CAS patients; D. CD14++CD16- monocyte/leukocytes in CAS patients. $* \mathrm{P}<0.05,{ }^{*} \mathrm{P}<0.01$.

A

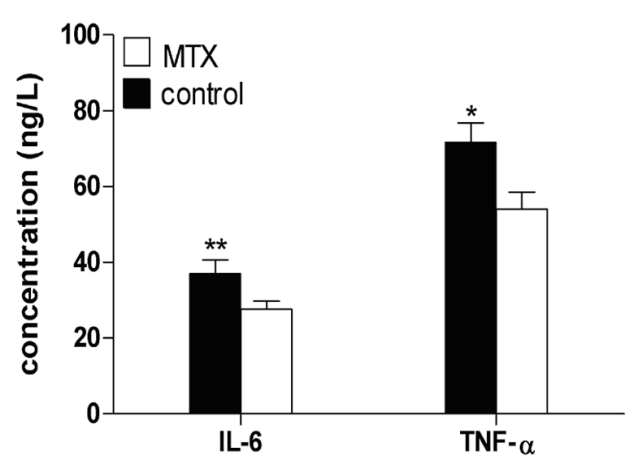

B

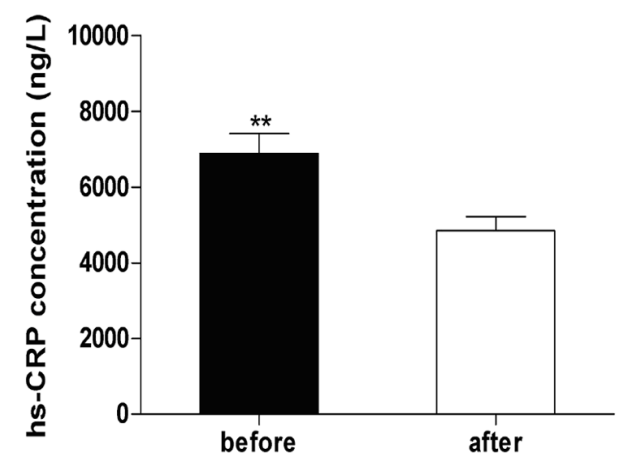

Figure 5. A. Concentrations of IL- 6 and TNF- $\alpha$ of CAS patients; B. Levels of hs-CRP level CAS patients. Control $=$ control group. $\mathrm{XMT}=$ Xuemaitong group.

\section{DISCUSSION}

TNF- $\alpha$, IL-6, and CRP are related to AS. When TNF- $\alpha$ acts on vascular endothelial cells, 
vascular injury and thrombosis occur (Miller et al., 2005). IL-6 plays an important role in the modulation of inflammation, host defense, and tissue damage. Additionally, it is crucial in the acute phase reaction and in CRP synthesis in liver cells. Furthermore, TNF- $\alpha$ is the main circulation material that connects the systemic immune response to local vascular injury. CRP is considered to be one of the most important inflammation markers of acute cardiovascular events. Studies have shown that CRP has direct effects on the proinflammatory response as it can directly influence the atherosclerotic lesion vessels by activating the complement system to accelerate the development of lesion inflammation and atherosclerosis. Makita et al. (2005) found that after adjusting for age and traditional risk factors, a correlation was observed between CRP and carotid artery plaques.

Monocyte-macrophages play a crucial role in the formation and development of atherosclerosis (Bobryshev, 2006; Swirski et al., 2006). Monocytes are a major source of atherosclerosis plaques in foam cells. Additionally, they are inflammatory cytokine-generating cells and secrete various matrix metalloproteinases to degrade the fiber composition in plaques, promoting the formation of vulnerable plaque. Monocytes can be divided into 2 subpopulations (i.e., $\mathrm{CD} 14++\mathrm{CD} 16-$ and $\mathrm{CD} 14+\mathrm{CD} 16+$ ) according to their varying expression levels of CD16. CD14++CD16- subpopulations are the major type of monocytes, while the CD14+ CD16+ subpopulation accounts for only $5-10 \%$ of monocytes. Compared with the CD14++ CD16- subpopulation, inflammatory chemotactic factors are present on the CD14+CD16+ cell membrane surfaces and can upregulate receptor expression (Tacke et al., 2007) and enhance the formation of TNF- $\alpha$ and IL-6 (Frankenberger et al., 1996; Belge et al., 2002; Ancuta et al., 2006). Belge et al. (2002) showed that CD14+CD16+ monocytes were the main source of TNF- $\alpha$. Schlitt et al. (2004) found that the expression of peripheral CD14+CD16+ monocytes was closely associated with the formation of TNF- $\alpha$. Additionally, high levels of vascular cell adhesion molecule-1 (ICAM - 1) ligands, CD11a and CD11c ligands, were observed in CD14+CD16+ monocytes, which may promote the adhesion of these cells to the endothelium and the migration of monocytes into target tissues (Graziano et al., 2003). Furthermore, Heine et al. (2008) proposed that CD14++CD16+ monocytes, but not total monocyte numbers, could be used to predict cardiovascular events in dialysis patients.

Previous studies indicated that Xuemaitong granules could induce a remarkable decrease in blood lipid levels, particularly triglycerides (Zhang et al., 2006, 2007). In this study, apoE-knockout mice were used to investigate the influence of Xuemaitong granules on GrlLow (equivalent to the CD14+CD16+ inflammation mononuclear cells). Lipid metabolism disorders and blood vessel wall damage are important factors in the pathogenesis of AS. In apoE-knockout mice, hypercholesterolemia may occur as the clearance of cholesterol was found to be reduced. Additionally, fiber plaques and composite plaques were formed, of which the distribution was similar to those observed in humans. Our results demonstrated that the monocyte to leukocyte ratio in the Xuemaitong group was significantly lower than that in the control group. We hypothesize that this was mainly caused by the GrlLow subset. Nevertheless, a significant decrease was noted in the Grlhigh subset (equivalent to the CD14+CD16+mononuclear cells). Compared with the control group, the levels of IL-6, TNF- $\alpha$, and hsCRP showed significant decreases after administration of Xuemaitong granules.

To investigate the effects of Xuemaitong on the pathogenesis of CAS, 100 CAS patients were selected. The proportion of monocytes and CD14+CD16+ monocytes was determined. After treatment, a significant decrease was observed in the proportion of peripheral blood monocytes among leukocytes. This was mainly caused by CD14+CD16+ monocytes. 
However, no remarkable decrease was noted in CD14+CD16- monocytes. Additionally, serum IL-6, TNF- $\alpha$, and hsCRP in the Xuemaitong group showed a significant decrease compared with the control group.

In conclusion, TCM Xuemaitong granules can reduce the secretion of inflammation factors by attenuating the formation of CD14+CD16+ monocytes. We propose that this medicine may be useful for treating carotid artery atherosclerosis.

\section{ACKNOWLEDGMENTS}

Research supported by Grants \#BK2010195 from the Council of Science and Technology, Jiangsu, China.

\section{REFERENCES}

Ancuta P, Wang J and Gabuzda D (2006). CD16+ monocytes produce IL-6, CCL2, and matrix metalloproteinase-9 upon interaction with CX3CL1-expressing endothelial cells. J. Leukoc. Biol. 80: 1156-1164.

Belge KU, Dayyani F, Horelt A, Siedlar M, et al. (2002). The proinflammatory CD14+CD16+DR++ monocytes are a major source of TNF. J. Immunol. 168: 3536-3542.

Berg KE, Ljungcrantz I, Andersson L, Bryngelsson C, et al. (2012). Elevated CD14++. Circ. Cardiovasc. Genet. 5: 122-131.

Bobryshev YV (2006). Monocyte recruitment and foam cell formation in atherosclerosis. Micron. 37: 208-222.

Frankenberger M, Sternsdorf T, Pechumer H, Pforte A, et al. (1996). Differential cytokine expression in human blood monocyte subpopulations: a polymerase chain reaction analysis. Blood 87: 373-377.

Fulda S, Strauss G, Meyer E and Debatin KM (2000). Functional CD95 ligand and CD95 death-inducing signaling complex in activation-induced cell death and doxorubicin-induced apoptosis in leukemic T cells. Blood 95: 301-308.

Graziano F, Humar B and Guilford P (2003). The role of the E-cadherin gene (CDH1) in diffuse gastric cancer susceptibility: from the laboratory to clinical practice. Ann. Oncol. 14: 1705-1713.

Heine GH, Ulrich C, Seibert E, Seiler S, et al. (2008). CD14(++)CD16+ monocytes but not total monocyte numbers predict cardiovascular events in dialysis patients. Kidney Int. 73: 622-629.

Imanishi T, Ikejima H, Tsujioka H, Kuroi A, et al. (2010). Association of monocyte subset counts with coronary fibrous cap thickness in patients with unstable angina pectoris. Atherosclerosis 212: 628-635.

Makita S, Nakamura M and Hiramori K (2005). The association of C-reactive protein levels with carotid intima-media complex thickness and plaque formation in the general population. Stroke 36: 2138-2142.

Merino A, Buendia P, Martin-Malo A, Aljama P, et al. (2011). Senescent CD14+CD16+ monocytes exhibit proinflammatory and proatherosclerotic activity. J. Immunol. 186: 1809-1815.

Miller AM, McPhaden AR, Preston A, Wadsworth RM, et al. (2005). TNF- $\alpha$ increases the inflammatory response to vascular balloon injury without accelerating neointimal formation. Atherosclerosis 179: 51-59.

Rogacev KS, Cremers B, Zawada AM, Seiler S, et al. (2012). CD14++CD16+ monocytes independently predict cardiovascular events: a cohort study of 951 patients referred for elective coronary angiography. J. Am. Coll. Cardiol. 60: 1512-1520.

Ross R (1999). Atherosclerosis-an inflammatory disease. N. Engl. J. Med. 340: 115-126.

Schlitt A, Heine GH, Blankenberg S, Espinola-Klein C, et al. (2004). CD14+CD16+ monocytes in coronary artery disease and their relationship to serum TNF- $\alpha$ levels. Thromb. Haemost. 92: 419-424.

Swirski FK, Pittet MJ, Kircher MF, Aikawa E, et al. (2006). Monocyte accumulation in mouse atherogenesis is progressive and proportional to extent of disease. Proc. Natl. Acad. Sci. U. S. A. 103: 10340-10345.

Tacke F, Alvarez D, Kaplan TJ, Jakubzick C, et al. (2007). Monocyte subsets differentially employ CCR2, CCR5, and CX3CR1 to accumulate within atherosclerotic plaques. J. Clin. Invest. 117: 185-194.

Zawada AM, Rogacev KS, Schirmer SH, Sester M, et al. (2012). Monocyte heterogeneity in human cardiovascular disease. Immunobiology 217: 1273-1284.

Zhang BX, Zeng XH and Zhang YM (2007). Empirical study of Xuemaitong granula on carotis artherosclerosis. Shanxi J. Traditional Chin. Med. 28: 1254-1256.

Zhang Q, Zhang BX, Jiang XX and Shi BW (2006). Clinical study on effect of Xuemaitong granule on cervical artherosclerosis. J. Nanjing Univ. Traditional Chin. Med. 22: 163-164. 